\title{
ON APPROXIMATE SOLUTIONS OF A CERTAIN HYPERBOLIC PARTIAL DIFFERENTIAL EQUATION
}

\author{
B. G. PACHPATTE
}

\begin{abstract}
In this paper we study approximate solutions of a certain hyperbolic partial differential equation with the given initial boundary conditions. A variant of a certain fundamental integral inequality with explicit estimate is used to establish the results. The discrete analogues of the main results are also given.
\end{abstract}

\section{Introduction}

Let $R^{n}$ denotes the real $n$-dimensional Euclidean space with appropriate norm denoted by |.|. We denote by $R_{+}=[0, \infty)$ the given subset of $R$, the set of real numbers. The partial derivatives of a function $z(x, y)$ for $x, y \in R_{+}$with respect to $x, y$ and $x y$ are denoted by $D_{1} z(x, y), D_{2} z(x, y)$ and $D_{2} D_{1} z(x, y)=D_{1} D_{2} z(x, y)$ and ' denotes the derivative. Let $C(A, B)$ denotes the class of continuous functions from the set $A$ to the set $B$. Recently, in [8] the author studied some basic qualitative properties of solutions of the initial boundary value problem (IBVP for short) for the hyperbolic equation

$$
D_{2} D_{1} u(x, y)=f\left(x, y, u(x, y), D_{1} u(x, y)\right) \text {, }
$$

with the given initial boundary conditions

$$
u(x .0)=\alpha(x), u(0, y)=\beta(y), u(0,0)=0,
$$

for $x, y \in R_{+}$, where $f \in C\left(R_{+}^{2} \times R^{n} \times R^{n}, R^{n}\right), \alpha, \beta \in C\left(R_{+}, R^{n}\right)$. In the literature there are many papers dealing with the qualitative properties of solutions of equations of the forms (1.1)-(1.2) by using different techniques, see $[1,2,3,10]$ and the references cited therein.

In the present paper, we offer the conditions for the error evaluation of approximate solutions of equation (1.1) by establishing new bounds and the convergence properties of solutions of approximate problems. The main tool employed in the analysis is based on the

Received November 05, 2009.

2000 Mathematics Subject Classification. 34K10, 35R10.

Key words and phrases. Approximate solutions, hyperbolic partial differential equation, integral inequality, explicit estimate, discrete analogues. 
application of a variant of an integral inequality with explicit estimate due to the present author given in [5]. The results on the discrete analogues of IBVP (1.1)-(1.2) are also given. A particular feature of our approach is that it present conditions under which we can offer some of the important qualitative properties of solutions of IBVP (1.1)-(1.2) in a simple and unified way.

\section{Main results}

We require the following variant of the integral inequality established by the present author and given in [5, Theorem 2.3.1, part $\left.a_{2}\right]$ (see also [8, p.42]).

Lemma 1. Let $u(x, y), a(x, y), b(x, y), e(x, y) \in C\left(R_{+}^{2}, R_{+}\right) ; e(x, y)$ be nondecreasing in each variable $x, y \in R_{+}$, and

$$
u(x, y) \leq e(x, y)+\int_{0}^{y} a(x, t) u(x, t) d t+\int_{0}^{x} \int_{0}^{y} b(s, t) u(s, t) d t d s,
$$

for $x, y \in R_{+}$, then

$$
u(x, y) \leq e(x, y) H(x, y) \exp \left(\int_{0}^{x} \int_{0}^{y} b(s, t) H(s, t) d t d s\right),
$$

for $x, y \in R_{+}$, where

$$
H(x, y)=\exp \left(\int_{0}^{y} a(x, \tau) d \tau\right)
$$

Let $u(x, y) \in C\left(R_{+}^{2}, R^{n}\right), D_{2} D_{1} u(x, y)$ exists and satisfies the inequality

$$
\left|D_{2} D_{1} u(x, y)-f\left(x, y, u(x, y), D_{1} u(x, y)\right)\right| \leq \varepsilon,
$$

for a given constant $\varepsilon \geq 0$, where it is supposed that (1.2) holds. Then we call $u(x, y)$ the $\varepsilon$ approximate solution with respect to the equation (1.1).

Our main result given in the following theorem estimates the difference between the two approximate solutions of equation (1.1).

Theorem 1. Suppose that the function $f$ in equation (1.1) satisfies the condition

$$
|f(x, y, z, w)-f(x, y, \bar{z}, \bar{w})| \leq p(x, y)[|z-\bar{z}|+|w-\bar{w}|],
$$

where $p \in C\left(R_{+}^{2}, R_{+}\right)$. Let $u_{i}(x, y)(i=1,2)$ for $x, y \in R_{+}$be respectively, $\varepsilon_{i}$-approximate solutions of equation (1.1) with

$$
u_{i}(x, 0)=\alpha_{i}(x), u_{i}(0, y)=\beta_{i}(y), u_{i}(0,0)=0
$$


where $\alpha_{i}, \beta_{i} \in C\left(R_{+}, R^{n}\right)$ and

$$
\left|\alpha_{1}(x)-\alpha_{2}(x)+\beta_{1}(y)-\beta_{2}(y)\right|+\left|\alpha_{1}^{\prime}(x)-\alpha_{2}^{\prime}(x)\right| \leq \delta,
$$

where $\delta \geq 0$ is a constant. Then

$$
\begin{aligned}
& \left|u_{1}(x, y)-u_{2}(x, y)\right|+\left|D_{1} u_{1}(x, y)-D_{1} u_{2}(x, y)\right| \\
& \quad \leq r(x, y) G(x, y) \exp \left(\int_{0}^{x} \int_{0}^{y} p(s, t) G(s, t) d t d s\right),
\end{aligned}
$$

for $x, y \in R_{+}$, where

$$
\begin{aligned}
r(x, y) & =\delta+\left(\varepsilon_{1}+\varepsilon_{2}\right)(y+x y) \\
G(x, y) & =\exp \left(\int_{0}^{y} p(x, \tau) d \tau\right) .
\end{aligned}
$$

Proof. Since $u_{i}(x, y)(i=1,2)$ for $x, y \in R_{+}$are respectively, $\varepsilon_{i}$-approximate solutions of equation (1.1) with (2.5), we have

$$
\left|D_{2} D_{1} u_{i}(x, y)-f\left(x, y, u_{i}(x, y), D_{1} u_{i}(x, y)\right)\right| \leq \varepsilon_{i}
$$

Keeping $x$ fixed in (2.10), setting $y=t$ and integrating both sides over $t$ from 0 to $y$, we observe that

$$
\begin{aligned}
\varepsilon_{i} y & \geq \int_{0}^{y}\left|D_{2} D_{1} u_{i}(x, t)-f\left(x, t, u_{i}(x, t), D_{1} u_{i}(x, t)\right)\right| d t \\
& \geq\left|\int_{0}^{y}\left\{D_{2} D_{1} u_{i}(x, t)-f\left(x, t, u_{i}(x, t), D_{1} u_{i}(x, t)\right)\right\} d t\right| \\
& =\left|D_{1} u_{i}(x, y)-\alpha_{i}^{\prime}(x)-\int_{0}^{y} f\left(x, t, u_{i}(x, t), D_{1} u_{i}(x, y)\right) d t\right|
\end{aligned}
$$

From (2.11) and using the elementary inequalities

$$
|v-z| \leq|v|+|z|,|v|-|z| \leq|v-z|
$$

we observe that

$$
\begin{aligned}
\left(\varepsilon_{1}+\varepsilon_{2}\right) y \geq & \left|D_{1} u_{1}(x, y)-\alpha_{1}^{\prime}(x)-\int_{0}^{y} f\left(x, t, u_{1}(x, t), D_{1} u_{1}(x, y)\right) d t\right| \\
& +\left|D_{1} u_{2}(x, y)-\alpha_{2}^{\prime}(x)-\int_{0}^{y} f\left(x, t, u_{2}(x, t), D_{1} u_{2}(x, y)\right) d t\right| \\
\geq & \mid\left\{D_{1} u_{1}(x, y)-\alpha_{1}^{\prime}(x)-\int_{0}^{y} f\left(x, t, u_{1}(x, t), D_{1} u_{1}(x, y)\right) d t\right\} \\
& -\left\{D_{1} u_{2}(x, y)-\alpha_{2}^{\prime}(x)-\int_{0}^{y} f\left(x, t, u_{2}(x, t), D_{1} u_{2}(x, y)\right) d t\right\} \mid
\end{aligned}
$$




$$
\begin{aligned}
\geq & \left|D_{1} u_{1}(x, y)-D_{1} u_{2}(x, y)\right|-\left|\alpha_{1}^{\prime}(x)-\alpha_{2}^{\prime}(x)\right| \\
& \quad-\int_{0}^{y}\left|f\left(x, t, u_{1}(x, t), D_{1} u_{1}(x, y)\right)-f\left(x, t, u_{2}(x, t), D_{1} u_{2}(x, y)\right)\right| d t .
\end{aligned}
$$

Keeping $y$ fixed in (2.11), setting $x=s$ and integrating both sides over $s$ from 0 to $x$, we have

$$
\begin{aligned}
\varepsilon_{i} x y & \geq \int_{0}^{x}\left|D_{1} u_{i}(s, y)-\alpha_{i}^{\prime}(s)-\int_{0}^{y} f\left(s, t, u_{i}(s, t), D_{1} u_{i}(s, y)\right) d t\right| d s \\
& \geq\left|\int_{0}^{x}\left\{D_{1} u_{i}(s, y)-\alpha_{i}^{\prime}(s)-\int_{0}^{y} f\left(s, t, u_{i}(s, t), D_{1} u_{i}(s, y)\right) d t\right\} d s\right| \\
& =\left|u_{i}(x, y)-\alpha_{i}(x)-\beta_{i}(y)-\int_{0}^{x} \int_{0}^{y} f\left(s, t, u_{i}(s, t), D_{1} u_{i}(s, y)\right) d t d s\right|
\end{aligned}
$$

From (2.14) and using the elementary inequalities in (2.12), we observe that

$$
\begin{aligned}
\left(\varepsilon_{1}+\varepsilon_{2}\right) x y \geq & \left|u_{1}(x, y)-\left[\alpha_{1}(x)+\beta_{1}(y)\right]-\int_{0}^{x} \int_{0}^{y} f\left(s, t, u_{1}(s, t), D_{1} u_{1}(s, t)\right) d t d s\right| \\
& +\left|u_{2}(x, y)-\left[\alpha_{2}(x)+\beta_{2}(y)\right]-\int_{0}^{x} \int_{0}^{y} f\left(s, t, u_{2}(s, t), D_{1} u_{2}(s, t)\right) d t d s\right| \\
\geq & \mid\left\{u_{1}(x, y)-\left[\alpha_{1}(x)+\beta_{1}(y)\right]-\int_{0}^{x} \int_{0}^{y} f\left(s, t, u_{1}(s, t), D_{1} u_{1}(s, t)\right) d t d s\right\} \\
& -\left\{u_{2}(x, y)-\left[\alpha_{2}(x)+\beta_{2}(y)\right]-\int_{0}^{x} \int_{0}^{y} f\left(s, t, u_{2}(s, t), D_{1} u_{2}(s, t)\right) d t d s\right\} \mid \\
\geq & \left|u_{1}(x, y)-u_{2}(x, y)\right|-\left|\left[\alpha_{1}(x)+\beta_{1}(y)\right]-\left[\alpha_{2}(x)+\beta_{2}(y)\right]\right| \\
& -\int_{0}^{x} \int_{0}^{y}\left|f\left(s, t, u_{1}(s, t), D_{1} u_{1}(s, t)\right)-f\left(s, t, u_{2}(s, t), D_{1} u_{2}(s, t)\right)\right| d t d s .(2.15)
\end{aligned}
$$

Let $w(x, y)=\left|u_{1}(x, y)-u_{2}(x, y)\right|+\left|D_{1} u_{1}(x, y)-D_{1} u_{2}(x, y)\right|$ for $x, y \in R_{+}$. From (2.13), (2.15) and using the hypotheses, we observe that

$$
\begin{aligned}
w(x, y) \leq & \left(\varepsilon_{1}+\varepsilon_{2}\right)(y+x y)+\left|\left[\alpha_{1}(x)+\beta_{1}(y)\right]-\left[\alpha_{2}(x)+\beta_{2}(y)\right]\right| \\
& +\left|\alpha_{1}^{\prime}(x)-\alpha_{2}^{\prime}(x)\right|+\int_{0}^{y} p(x, t) w(x, t) d t+\int_{0}^{x} \int_{0}^{y} p(s, t) w(s, t) d t d s \\
\leq & r(x, y)+\int_{0}^{y} p(x, t) w(x, t) d t+\int_{0}^{x} \int_{0}^{y} p(s, t) w(s, t) d t d s,
\end{aligned}
$$

where $r(x, y)$ is given by (2.8). Clearly $r(x, y)$ is nonnegative and nondecreasing in each variable $x, y \in R_{+}$. Now an application of Lemma 1 to (2.16) yields (2.7).

Remark 1. We note that the estimate obtained in (2.7) yields, not only the bound on the difference between the two approximate solutions of equation (1.1) with (2.5), but also the bound on the difference between their derivatives with respect to the first variable. If $u_{1}(x, y)$ is a solution of equation (1.1) with (2.5) (when $i=1$ ), then we have $\varepsilon_{1}=0$ and from (2.7) we see that $u_{2}(x, y) \rightarrow u_{1}(x, y)$ as $\varepsilon_{2} \rightarrow 0$ and $\delta \rightarrow 0$. Moreover, if we put $(i) \varepsilon_{1}=\varepsilon_{2}=0$ and 
$\alpha_{1}(x)=\alpha_{2}(x), \beta_{1}(y)=\beta_{2}(y)$, for $x, y \in R_{+}$in (2.7), then the uniqueness of solutions of equation (1.1) is established and ( $i$ i $) \varepsilon_{1}=\varepsilon_{2}=0$ in (2.7), then we get the bound which shows the dependency of solutions of equation (1.1) on given initial boundary values.

Now consider the IBVP (1.1)-(1.2) together with the following IBVP:

$$
\begin{aligned}
& D_{2} D_{1} v(x, y)=\bar{f}\left(x, y, v(x, y), D_{1} v(x, y)\right), \\
& v(x, 0)=\bar{\alpha}(x), v(0, y)=\bar{\beta}(y), v(0,0)=0,
\end{aligned}
$$

where $\bar{f} \in C\left(R_{+}^{2} \times R^{n} \times R^{n}, R^{n}\right) ; \bar{\alpha}, \bar{\beta} \in C\left(R_{+}, R^{n}\right)$.

In the next theorem we provide conditions concerning the closeness of solutions of IBVP (1.1)-(1.2) and IBVP (2.17)-(2.18).

Theorem 2. Suppose that the function $f$ in equation (1.1) satisfies the condition (2.4) and there exist constants $\bar{\varepsilon} \geq 0, \bar{\delta} \geq 0$ such that

$$
\begin{array}{r}
|f(x, y, z, w)-\bar{f}(x, y, z, w)| \leq \bar{\varepsilon}, \\
|\alpha(x)-\bar{\alpha}(x)+\beta(y)-\bar{\beta}(y)|+\left|\alpha^{\prime}(x)-\bar{\alpha}^{\prime}(x)\right| \leq \bar{\delta},
\end{array}
$$

where $f, \alpha, \beta$ and $\bar{f}, \bar{\alpha}, \bar{\beta}$ are as in IBVP (1.1)-(1.2) and IBVP (2.17)-(2.18). Let $u(x, y)$ and $v(x, y)$ be respectively the solutions of IBVP (1.1)-(1.2) and IBVP (2.17)-(2.18) for $x, y \in R_{+}$. Then

$$
\begin{aligned}
& |u(x, y)-v(x, y)|+\left|D_{1} u(x, y)-D_{1} v(x, y)\right| \\
& \quad \leq q(x, y) G(x, y) \exp \left(\int_{0}^{x} \int_{0}^{y} p(s, t) G(s, t) d t d s\right),
\end{aligned}
$$

for $x, y \in R_{+}$, where

$$
q(x, y)=\bar{\delta}+\bar{\varepsilon}(y+x y)
$$

and $G(x, y)$ is given by (2.9).

Proof. Let $z(x, y)=|u(x, y)-v(x, y)|+\left|D_{1} u(x, y)-D_{1} v(x, y)\right|$ for $x, y \in R_{+}$. Using the facts that $u(x, y), v(x, y)$ are the solutions of IBVP (1.1)-(1.2), IBVP (2.17)-(2.18) and hypotheses, we observe that

$$
\begin{aligned}
z(x, y) \leq & |\alpha(x)-\bar{\alpha}(x)+\beta(y)-\bar{\beta}(y)|+\left|\alpha^{\prime}(x)-\bar{\alpha}^{\prime}(x)\right| \\
& +\int_{0}^{y}\left|f\left(x, t, u(x, t), D_{1} u(x, t)\right)-f\left(x, t, v(x, t), D_{1} v(x, t)\right)\right| d t \\
& +\int_{0}^{y}\left|f\left(x, t, v(x, t), D_{1} v(x, t)\right)-\bar{f}\left(x, t, v(x, t), D_{1} v(x, t)\right)\right| d t
\end{aligned}
$$




$$
\begin{aligned}
& +\int_{0}^{x} \int_{0}^{y}\left|f\left(s, t, u(s, t), D_{1} u(s, t)\right)-f\left(s, t, v(s, t), D_{1} v(s, t)\right)\right| d t d s \\
& +\int_{0}^{x} \int_{0}^{y}\left|f\left(s, t, v(s, t), D_{1} v(s, t)\right)-\bar{f}\left(s, t, v(s, t), D_{1} v(s, t)\right)\right| d t d s \\
\leq & \bar{\delta}+\int_{0}^{y} p(x, t) z(x, t) d t+\int_{0}^{y} \bar{\varepsilon} d t+\int_{0}^{x} \int_{0}^{y} p(s, t) z(s, t) d t d s+\int_{0}^{x} \int_{0}^{y} \bar{\varepsilon} d t d s \\
= & q(x, y)+\int_{0}^{y} p(x, t) z(x, t) d t+\int_{0}^{x} \int_{0}^{y} p(s, t) z(s, t) d t d s,
\end{aligned}
$$

where $q(x, y)$ is given by (2.22). Clearly $q(x, y)$ is nonnegative and nondecreasing in each variable $x, y \in R_{+}$. Now an application of Lemma 1 to (2.23) yields (2.21).

Remark 2. We note that the result given in Theorem 2 relates the solutions of IBVP (1.1)-(1.2) and IBVP (2.17) - (2.18) in the sence that if $f$ is close to $\bar{f} ; \alpha(x), \beta(y)$ are close to $\bar{\alpha}(x), \bar{\beta}(y)$ and $\alpha^{\prime}(x)$ is close to $\bar{\alpha}^{\prime}(x)$ for $x, y \in R_{+}$, then the solutions of IBVP (1.1) - (1.2) and IBVP (2.17)-(2.18) are also close together.

Next, consider the IBVP (1.1)-(1.2) and sequence of IBVPs:

$$
\begin{aligned}
D_{2} D_{1} u(x, y) & =f_{k}\left(x, y, u(x, y), D_{1} u(x, y)\right), \\
u(x, 0) & =\alpha_{k}(x), u(0, y)=\beta_{k}(y), u(0,0)=0,
\end{aligned}
$$

for $x, y \in R_{+}$and $k=1,2, \ldots$, where $f_{k} \in C\left(R_{+}^{2} \times R^{n} \times R^{n}, R^{n}\right), \alpha_{k}, \beta_{k} \in C\left(R_{+}, R^{n}\right)$.

As a consequence of Theorem 2, the following corollary holds.

Corollary. Suppose that the function $f$ in equation (1.1) satisfies the condition (2.4) and there exist constants $\bar{\varepsilon}_{k} \geq 0, \bar{\delta}_{k} \geq 0$ such that

$$
\begin{array}{r}
\left|f(x, y, z, w)-f_{k}(x, y, z, w)\right| \leq \bar{\varepsilon}_{k}, \\
\left|\alpha_{k}(x)-\alpha(x)+\beta_{k}(y)-\beta(y)\right|+\left|\alpha_{k}^{\prime}(x)-\alpha^{\prime}(x)\right| \leq \bar{\delta}_{k},
\end{array}
$$

with $\bar{\varepsilon}_{k} \rightarrow 0$ and $\bar{\delta}_{k} \rightarrow 0$ as $k \rightarrow \infty$, where $f, \alpha, \beta$ and $f_{k}, \alpha_{k}, \beta_{k}$ are as in IBVP (1.1)-(1.2) and IBVPS (2.24)-(2.25). If $u_{k}(x, y)(k=1,2, \ldots)$ and $u(x, y)$ are respectively the solutions of IBVPS (2.24)-(2.25) and IBVP (1.1)-(1.2) for $x, y \in R_{+}$, then $u_{k}(x, y) \rightarrow u(x, y)$ for $x, y \in R_{+}$as $k \rightarrow \infty$.

Proof. For $k=1,2, \ldots$, the conditions of Theorem 2 hold. As an application of Theorem 2 yields

$$
\left|u_{k}(x, y)-u(x, y)\right| \leq \bar{q}(x, y) G(x, y) \exp \left(\int_{0}^{x} \int_{0}^{y} p(s, t) G(s, t) d t d s\right),
$$

for $x, y \in R_{+}$and $k=1,2, \ldots$, where

$$
\bar{q}(x, y)=\bar{\delta}_{k}+\bar{\varepsilon}_{k}(y+x y) .
$$

The required result follows from (2.28). 
Remark 3. We note that the Corollary provides sufficient conditions that ensures, solutions of IBVPs (2.24) - (2.25) will converge to the solution of IBVP (1.1)-(1.2).

\section{Discrete analogues}

Let $N_{0}=\{0,1,2, \ldots\}$ be a given subset of $R$ and $D(A, B)$, denotes the class of discrete functions from the set $A$ to the set $B$. For functions $z(m)$ and $w(m, n)$ for $m, n \in N_{0}$, we define the operators $\Delta, \Delta_{1}, \Delta_{2}$ by $\Delta z(m)=z(m+1)-z(m), \Delta_{1} w(m, n)=w(m+1, n)-w(m, n)$, $\Delta_{2} w(m, n)=w(m, n+1)-w(m, n)$, and $\Delta_{2} \Delta_{1} w(m, n)=\Delta_{2}\left(\Delta_{1} w(m, n)\right)$. We use the usual conventions that empty sums and products are taken to be 0 and 1 respectively. We now explore in brief our idea to obtain results similar to those given above concerning the discrete analogue of IBVP (1.1)-(1.2) which can be written as

$$
\begin{aligned}
\Delta_{2} \Delta_{1} u(m, n) & =f\left(m, n, u(m, n), \Delta_{1} u(m, n)\right), \\
u(m, 0) & =\alpha(m), u(0, n)=\beta(n), u(0,0)=0,
\end{aligned}
$$

for $m, n \in N_{0}$, where $f \in D\left(N_{0}^{2} \times R^{n} \times R^{n}, R^{n}\right), \alpha, \beta \in D\left(N_{0}, R^{n}\right)$. In this section we formulate in brief the discrete analogues of Lemma 1 and Theorems 1 and 2, whose proofs can be completed by following the idea used to prove the above results and closely looking at the similar results given in $[6,9]$.

Let $u \in D\left(N_{0}^{2}, R^{n}\right)$ and $\Delta_{2} \Delta_{1} u(m, n)$ for $m, n \in N_{0}$ exists and satisfies the inequality

$$
\left|\Delta_{2} \Delta_{1} u(m, n)-f\left(m, n, u(m, n), \Delta_{1} u(m, n)\right)\right| \leq \varepsilon,
$$

for a given constant $\varepsilon \geq 0$, where it is supposed that (3.2) holds. Then we call $u(m, n)$ an $\varepsilon$ approximate solution to the equation (3.1).

Lemma 2. Let $u(m, n), a(m, n), b(m, n), e(m, n) \in D\left(N_{0}^{2}, R_{+}\right) ; e(m, n)$ be nondecreasing in each variable $m, n \in N_{0}$ and

$$
u(m, n) \leq e(m, n)+\sum_{t=0}^{n-1} a(m, t) u(m, t)+\sum_{s=0}^{m-1} \sum_{t=0}^{n-1} b(s, t) u(s, t),
$$

for $m, n \in N_{0}$, then

$$
u(m, n) \leq e(m, n) \bar{H}(m, n) \prod_{s=0}^{m-1}\left[1+\sum_{t=0}^{n-1} b(s, t) \bar{H}(s, t)\right],
$$

for $m, n \in N_{0}$, where

$$
\bar{H}(m, n)=\prod_{t_{1}=0}^{n-1}\left[1+a\left(m, t_{1}\right)\right] .
$$


Theorem 3. Suppose that the function $f$ in equation (1.1) satisfies the condition

$$
|f(m, n, z, w)-f(m, n, \bar{z}, \bar{w})| \leq p(m, n)[|z-\bar{z}|+|w-\bar{w}|],
$$

where $p \in D\left(N_{0}^{2}, R_{+}\right)$. Let $u_{i}(m, n)(i=1,2)$ for $m, n \in N_{0}$ be respectively $\varepsilon_{i}$-approximate solutions of equation (3.1) with

$$
u_{i}(m, 0)=\alpha_{i}(m), u_{i}(0, n)=\beta_{i}(n), u_{i}(0,0)=0,
$$

where $\alpha_{i}, \beta_{i} \in D\left(N_{0}, R^{n}\right)$ and

$$
\left|\alpha_{1}(m)-\alpha_{2}(m)+\beta_{1}(n)-\beta_{2}(n)\right|+\left|\Delta \alpha_{1}(m)-\Delta \alpha_{2}(m)\right| \leq \delta,
$$

where $\delta \geq 0$ is a constant. Then

$$
\begin{aligned}
& \left|u_{1}(m, n)-u_{2}(m, n)\right|+\left|\Delta_{1} u_{1}(m, n)-\Delta_{1} u_{2}(m, n)\right| \\
& \leq a(m, n) \bar{G}(m, n) \prod_{s=0}^{m-1}\left[1+\sum_{t=0}^{n-1} p(s, t) \bar{G}(s, t)\right],
\end{aligned}
$$

for $m, n \in N_{0}$, where

$$
a(m, n)=\delta+\left(\varepsilon_{1}+\varepsilon_{2}\right)(n+m n),
$$

and

$$
\bar{G}(m, n)=\prod_{t_{1}=0}^{n-1}\left[1+p\left(m, t_{1}\right)\right]
$$

Next, we consider the IBVP (3.1)-(3.2) together with the following IBVP:

$$
\begin{aligned}
& \Delta_{2} \Delta_{1} v(m, n)=\bar{f}\left(m, n, v(m, n), \Delta_{1} v(m, n)\right), \\
& v(m, 0)=\bar{\alpha}(m), \quad v(0, n)=\bar{\beta}(n), \quad v(0,0)=0,
\end{aligned}
$$

where $\bar{f} \in D\left(N_{0}^{2} \times R^{n} \times R^{n}, R^{n}\right), \bar{\alpha}, \bar{\beta} \in D\left(N_{0}, R^{n}\right)$.

Theorem 4. Suppose that the function $f$ in equation (3.1) satisfies the condition (3.7) and there esist constants $\bar{\varepsilon} \geq 0, \bar{\delta} \geq 0$ such that

$$
\begin{array}{r}
|f(m, n, z, w)-\bar{f}(m, n, z, w)| \leq \bar{\varepsilon}, \\
|\alpha(m)-\bar{\alpha}(m)+\beta(n)-\bar{\beta}(n)|+|\Delta \alpha(m)-\Delta \bar{\alpha}(m)| \leq \bar{\delta},
\end{array}
$$

where $f, \alpha, \beta$ and $\bar{f}, \bar{\alpha}, \bar{\beta}$ are as in IBVP (3.1)-(3.2) and IBVP (3.14)-(3.15). Let $u(m, n)$ and $v(m, n)$ be respectively the solutions of IBVP (3.1)-(3.2) and IBVP (3.14)-(3.15) for $m, n \in N_{0}$. Then

$$
|u(m, n)-v(m, n)|+\left|\Delta_{1} u(m, n)-\Delta_{1} v(m, n)\right|
$$




$$
\leq b(m, n) \bar{G}(m, n) \prod_{s=0}^{m-1}\left[1+\sum_{t=0}^{n-1} p(s, t) \bar{G}(s, t)\right] \text {, }
$$

for $m, n \in N_{0}$, where

$$
b(m, n)=\bar{\delta}+\bar{\varepsilon}(n+m n),
$$

and $\bar{G}(m, n)$ is given by (3.13).

Remark 4. We note that the idea used in this paper can be extended to study the approximate solutions of the following hyperbolic equations

$$
\begin{aligned}
& D_{2} D_{1} u(x, y)=f\left(x, y, u(x, y), D_{2} u(x, y)\right), \\
& D_{2} D_{1} u(x, y)=f\left(x, y, u(x, y), D_{1} u(x, y), D_{2} D_{1} u(x, y)\right), \\
& D_{2} D_{1} u(x, y)=f\left(x, y, u(x, y), D_{2} u(x, y), D_{2} D_{1} u(x, y)\right), \\
& D_{2} D_{1} u(x, y)=f\left(x, y, u(x, y), D_{2} D_{1} u(x, y), E u(x, y)\right),
\end{aligned}
$$

with

$$
E u(x, y)=\int_{0}^{x} \int_{0}^{y} g\left(x, y, \sigma, \tau, u(\sigma, \tau), D_{2} D_{1} u(\sigma, \tau)\right) d \tau d \sigma,
$$

for $x, y \in R_{+}$, with the given initial boundary conditions in (1.2), and their discrete analogues by making use of the suitable variants of the inequalities given in Lemmas 1 and 2 (see also $[4,5,6,7])$. The details of the formulations of such results are very close to those given above with suitable modifications. Here we omit the details.

\section{References}

[1] A. Alexiewicz and W. Orlicz, Some remarks on the existence and uniqueness of solutions of the hyperbolic equation $\frac{\partial^{2} z}{\partial x \partial y}=f\left(x, y, z, \frac{\partial z}{\partial x}, \frac{\partial z}{\partial y}\right)$, Studia Math., 15(1956), 201-215.

[2] L. Byszewski, Existence and uniqueness of solutions of nonlocal problems for hyperbolic equation $u_{x t}=$ $F\left(x, t, u, u_{x}\right)$, J. Appl. Math. Stochastic Analysis, 3(1990), 163-168.

[3] J. Kisynski and A. Pelczar, Comparison of solutions and successive approximations in the theory of equation $\frac{\partial^{2} z}{\partial x \partial y}=f\left(x, y, z, \frac{\partial z}{\partial x}, \frac{\partial z}{\partial y}\right)$, Dissertations Mathematicae, 76(1970), 1-77.

[4] B. G. Pachpatte, Inequalities for Finite Difference Equations, Marcel Dekker Inc., New York, 2002.

[5] B. G. Pachpatte, Integral and Finite Difference Inequalities and Applications, North-Holland Mathematics Studies, Vol. 205, Elsevier Science B.V., Amsterdam, 2006

[6] B. G. Pachpatte, On a certain finite difference equation in two independent variables, Bul. Inst. Polit. Iaşi Univ. Tech. "Gh.Asachi", Ser. Mat. Meca. Teor. Fiz., LIII(LVII) (2007), 35-44.

[7] B. G. Pachpatte, On neutral type hyperbolic integrodifferential equation, Fasc. Math. Nr., 40(2008), 57-69.

[8] 8. B.G. Pachpatte, On a certain hyperbolic partial differential equation, Tamusi Oxford Jour. Math. Sci., 25(2009), 39-54.

[9] B. G. Pachpatte, Error evaluation of approximate solutions for sum-difference equations in two variables, Elect. Jour. Diff. Eqs., 2009(2009), 1-8.

[10] W. Walter, Differential and Integral inequalities, Springer-Verlag, Berlin, New York, 1970. 
57 Shri Niketan Colony, Near Abhinay Talkies, Aurangabad 431001 (Maharashtra) India.

E-mail: bgpachpatte@gmail.com 